\title{
Cycles de vie de quelques Plécoptères (Insecta) d'un ruisseau temporaire du Liban'
}

\author{
N.J. Alouf2
}

Mots clés : Plécoptères, Liban, ruissseaux temporaires, développement larvaire.

Le développement Jarvaire des 4 espèces de Plécoptères Capnia ? arensi Zhiltzova, Brachyptera galeata Koponen, Prota nemu ra libanocypria Zwick et Isoperla libanica Aubert est étudié. Ces espèces colonisent un ruisseau temporaire du Liban. $C$. ? arensi est une espèce hivernale, $B$. galeata et $P$. libanocypria sont printanières, $I$. libanica est printanière tardive.

Life cycles of some Pleceptera (Insecta) from a temporary stream of Lebanon.

Keywords : Plecoptera, Lebanon, temporary streams, larval development.

The larval development of 4 species of Plecoptera Capnia ? arensi Zhiltzova, Brachyptera galeata Koponen, Protonemura libarocypria Zwick and Isoperla libanica Aubert is studied. These species colonize a temporary stream of Lebanon. C.? arensi is a winter species, $B$. galeata and $P$. libanocypria are vernal, I. libanica is late vemal.

Les Plécoptères Brachyptera galeata, Protonemura libanocypria, Isoperla libanica et Capnia? arensi colonisent un affluent du haut bassin du Litani (Alouf 1983 a). Ces espèces ont une distribution proche orientale et leur développernent larvaire n'est pas connu. En 1979 et 1980, les récoltes d'un nombie suffisant de larves et d'adultes nous ont permis d'étudier leur cycle de vie.

\section{Matériel et méthodes}

La zone montagnarde du Nahr Qab Ilias (Station I in Alouf 1983 a), connue sous le nom de Wadi Dalam, est à régime temporaire. Au printemps 1978, des récoltes d'imagos et à l'automne 1978, 1979 et 1980 des récoltes de larves et d'adultes ont été effectuées. Des prélèvements benthiques proviennent de deux stations : la première située dans un ruisselet issu d'une exsurgence temporaire $\left(33^{\circ} 39^{\prime} 03^{\prime}\right.$ ' $\mathbf{L N}$, $35^{\circ} 47^{\prime} \mathrm{LE}$ ) et la seconde dans le cours d'eau principal du ruisseau une centaine de mètres plus bas.

1. Travail subventionné par le C.N.R.S. Liban : Projet $\mathbf{N}^{\circ} 50.008$.

2. Université Libanaise, Faculté des Sciences. Correspondance Nicolas J. Alouf, Quartier_Barbara, Zahlé, Liban.
Le filet utilisé est à bords droits avec un vide de maille de 0,3 mm. En 1978, la chasse aux adultes a eu ljeu chaque semaine, en 1979, chaque deux semaines et en 1989 à intervalles plus irréguliers en fonction de la période de vol, toujours pendant une durée de 30 minutes à une heure (Tableau I). La largeur de la capsule céphalique, repère de la crois sance larvaire, a été mesurée au micromètre oculaire. Les larves sont séparées en classes de $0,1 \mathrm{~mm}$.

\section{Développement larvaire et périodes de vol}

Capnia ? arensi Zhiltzova, 19643

Les larvules font leur apparition dès la minovembre à la suite de la remise en eau du cours. On rencontre des larves jusqu'en février (fig. 1). Cette espèce n'a pas de préférence pour le ruisselet ou pour le cours principal (Tableau II). Le vol commence entre fin-janvier et mi-février. Le vol le plus précoce a été observé le 28 janvier 1980 . Les mâles

\footnotetext{
3. Le développement plus important des protubérances, dès le $7^{*}$ tergite, jơtnt à d'aut res caractérist iques morphologiques, aussi bien cher le mâle que chez la femelle, nous incitent a assimiler de façon incertaine cette population à C. arensi.
} 
Tableau I. Dates de capture et répartition des Plécoptères mâles et femelles.

* : imagos récoltés à l'aval de la région prospectée

** : récolte pendant un orage.

\begin{tabular}{|c|c|c|c|c|c|c|c|c|}
\hline & \multirow[t]{2}{*}{ c. } & libanica & \multicolumn{2}{|c|}{ B. galeata } & \multicolumn{2}{|c|}{ P. libanocypria } & \multicolumn{2}{|c|}{ I. libanica } \\
\hline & & $q$ & $\vec{\sigma}$ & $q$ & $\sigma$ & q & $\sigma$ & $q$ \\
\hline $\begin{array}{r}16 / 2 / 78 \\
2 / 3 / 78 \\
21 / 3 / 78 \\
8 / 4 / 78 \\
18 / 4 / 78 \\
26 / 4 / 78 \\
2 / 5 / 78 \\
9 / 5 / 78 \\
16 / 5 / 78 \\
23 / 5 / 78 \\
30 / 5 / 78 \\
6 / 6 / 78 \\
14 / 6 / 78\end{array}$ & $1 \star$ & 13 & $\begin{array}{l}1 \text { * } \\
1 \text { * } \\
77^{2} \\
12 \\
3 \\
3 \\
1\end{array}$ & $\begin{array}{r}61 \\
27 \\
11 \\
7\end{array}$ & $\begin{array}{r}19 \\
85 \\
85 \\
161 \\
98 \\
23 \\
6\end{array}$ & $\begin{array}{r}20 \\
62 \\
57 \\
79 \\
65 \\
11 \\
5\end{array}$ & $\begin{array}{r}2 \\
32 \\
57 \\
52 \\
34\end{array}$ & $\begin{array}{r}4 \\
20 \\
41 \\
79 \\
47 \\
4\end{array}$ \\
\hline $\begin{array}{r}15 / 2 / 79 \\
3 / 3 / 79 \\
17 / 3 / 79 \\
30 / 3 / 79 \\
13 / 4 / 79 \\
27 / 4 / 79 \\
11 / 5 / 79\end{array}$ & $\begin{array}{r}13 \\
5 \\
2\end{array}$ & $\begin{array}{l}2 \\
2 \\
2 \\
5\end{array}$ & $\begin{array}{r}3 \\
14 \\
62 \\
34 \\
19\end{array}$ & $\begin{array}{l}22 \\
64 \\
34 \\
32\end{array}$ & $\begin{array}{r}3 \\
12 \\
74 \\
32 \\
38 \\
33\end{array}$ & $\begin{array}{r}7 \\
44 \\
33 \\
26 \\
15\end{array}$ & $\uparrow$ & $\begin{array}{l}1 \\
1\end{array}$ \\
\hline $\begin{array}{c}20 / 1 / 80 \\
13 / 2 / 80 \\
11 / 3 / 80 \\
2 / 4 / 80^{*} \\
22 / 4 / 80 \\
30 / 4 / 80 \\
8 / 5 / 80 \\
22 / 5 / 80 \\
29 / 5 / 80 \\
12 / 6 / 80\end{array}$ & $\begin{array}{l}18 \\
12\end{array}$ & $\begin{array}{l}2 \\
3 \\
2\end{array}$ & $\begin{array}{r}15 \\
6 \\
10 \\
3 \\
1\end{array}$ & $\begin{array}{r}1 \\
10 \\
3 \\
20 \\
3 \\
2\end{array}$ & $\begin{array}{r}25 \\
3 \\
27 \\
37 \\
36 \\
44 \\
7\end{array}$ & $\begin{array}{r}7 \\
1 \\
26 \\
22 \\
34 \\
57 \\
6\end{array}$ & $\begin{array}{r}2 \\
11 \\
1\end{array}$ & $\begin{array}{r}2 \\
17 \\
5\end{array}$ \\
\hline
\end{tabular}

dominent en février et pendant la première moitié de mars. Les femelles ont une importante longévité : elles sont présentes en avril, soit un mois et demi après la disparition des larves. La période de vol dure 9 semaines (fig. 2). La durée de vie des femelles doit être d'un mois. C'est une espèce hivernale.

\section{Brachyptera galeata Koponen, 1949}

Cette espèce a une préférence pour le ruisselet (Tableau II). On rencontre des larves de janvier à avril inclus (fig. 1). Les premiers adultes ont été observés le 13 février 1980, les derniers pendant la première décade de mai. La période de vol s'étale sur trois mois environ. Les imagos se rencontrent surtout sur les troncs d'arbres. C'est une espece printanière (Berthélemy \& Dia 1982).

\section{Protonemura libanocypria Zwick, 1978}

Tout comme $B$. galeata, cette espèce a une préférence marquée pour le ruisselet (Tableau II). Les larvules font leur apparition en janvier. On rencontre des larves jusqu'à la fin avril (fig. 1). La période de vol s'étend de mars à mai. Le vol le plus précoce a été observé le 3 mars 1979, le dernier fin mai. La periode de vol dure donc trois mois. Les imagos ont été récoltés sur le tronc des arbres. C'est une espèce printanière. 
Isoperla libanica Aubert, 1964

Cette espèce a une préférence marquée pour le cours principal (Tableau II). Les premières larves, déjà âgées, ont été récoltées mi-février et les dernières en mai ( $f i g .1$ ). La période de vol dure 6 à 7 semaines : elle couvre le mois de mai et, éventuellement, le mois de juin si les conditions sont favorables, par exemple, quand le cours d'eau est encore inondé. Les imagos, de couleur verte, ont été récoltés sur l'herbe en pleine croissance à cet te époque de l'année. C'est une espèce printanière tardive.

\section{Discussion}

Légier (1979) résume l'essentiel de nos connaissances sur le développement préimaginal des insectes aquatiques dans les ruisseaux temporaires. Plusieurs mécanismes permettent aux organismes de surmonter la phase d'assèchement : soit par quiescence larvaire, soit par migration des larves vers le substrat profond toujours humide. On peut ajouter à ces mécanismes l'allongement de la durée d'incubation des oufs comme c'est le cas chez Perlodes microcephalus où les ceufs peuvent éclore soit 2 à 5 mois après la ponte, soit jusqu'à un an plus tard (Berthélemy 1979).
Au cas ou le développement de $C$. ? arensi serait comparable à celui de $C$. bifrons les oeufs doivent éclore juste après la ponte, les larvules entrent en diapause parce que la photopériode est positive. La remise en eau en novembre, concomitante de la photopériode négative, déclenche la reprise de la vie active. Quatre mois sont suffisants pour effectuer la mue imaginale.

L'éclosion des premières pontes printanières précoces de $B$. galeata et de $P$. libanocypria aurait lieu dès la remise en eau en automne. Elle serait suivie par un enfouissement des larvules. Les larves remontent en surface en janvier. L'éclosion des pontes tardives aurait lieu à cette époque. Les larves ont besoin de trois à quatre mois pour effectuer leur mue imaginale. Le cycle de vie de ces espèces ressemblerait à celui de Nemoura cinerea dans le sud de la France (Légier 1979).

Le vol de I. libanica a lieu juste avant l'assèchement du cours d'eau. Les femelles, du moins les dernières à effectuer leur vol, pondent dans les flaques d'eau persistantes. La période d'incubation des œufs est nécessairement supérieure à la phase sèche pour que le processus d'éclosion se déclenche dès la remise en eau. Ce phénomène d'incubation a été

Tableau II. Répartition annuelle des larves de Plécoptères à Wadi Dalam.

$\mathbf{R}$ : ruisseau issu de l'exsurgence temporaire :

C: cours principal.

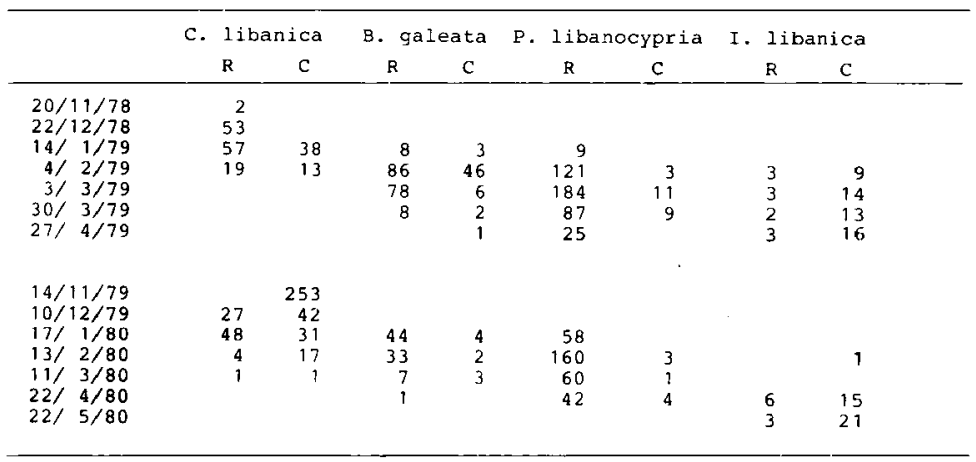


décrit pour certaines espèces européennes (Saltveit et al. 1984). Les larvules migrent en profondeur pour ne remonter qu'en février. Le développement larvaire est rapide et la mue imaginale survient en mai. Cette migration serait un moyen de lutte contre l'ensoleillement excessif dans cette région et également une adaptation aux mauvaises conditions écologiques. Ce phénomène d'enfouissement des premiers stades de développement a déjà été observé chez les jeunes Gammares de Shtaura (Alouf 1983 b).

\section{Conclusion}

La sècheresse estivale élimine tout insecte aquatique ayant besoin de plus d'un semestre de vie active. Le développement préimaginal de $C$ ? arensi à Wadi Dalam est automno-hivernal, celui des autres Plécoptères est essentiellement hivernal (fig. 1). La sex-ratio des populations imaginales est initialement protandre, elle devient protogyne à la fin de la période de vol, excepté chez $P$. libanocypria où elle reste protandre (Tableau I, fig. 2).

Les dates de capture de I. libanica signalées par Aubert (1984), de P. libanocypria par Zwick (1978), de B. galeata, P. libanocypria, I. libanica par Berthélemy \& Dia (1982) sont fragmentaires. Les récoltes obtenues a Wadi Dalam permettent de mieux cerner les périodes de vol de ces espèces, du moins pour ce qui concerne la région prospectée. Les durées

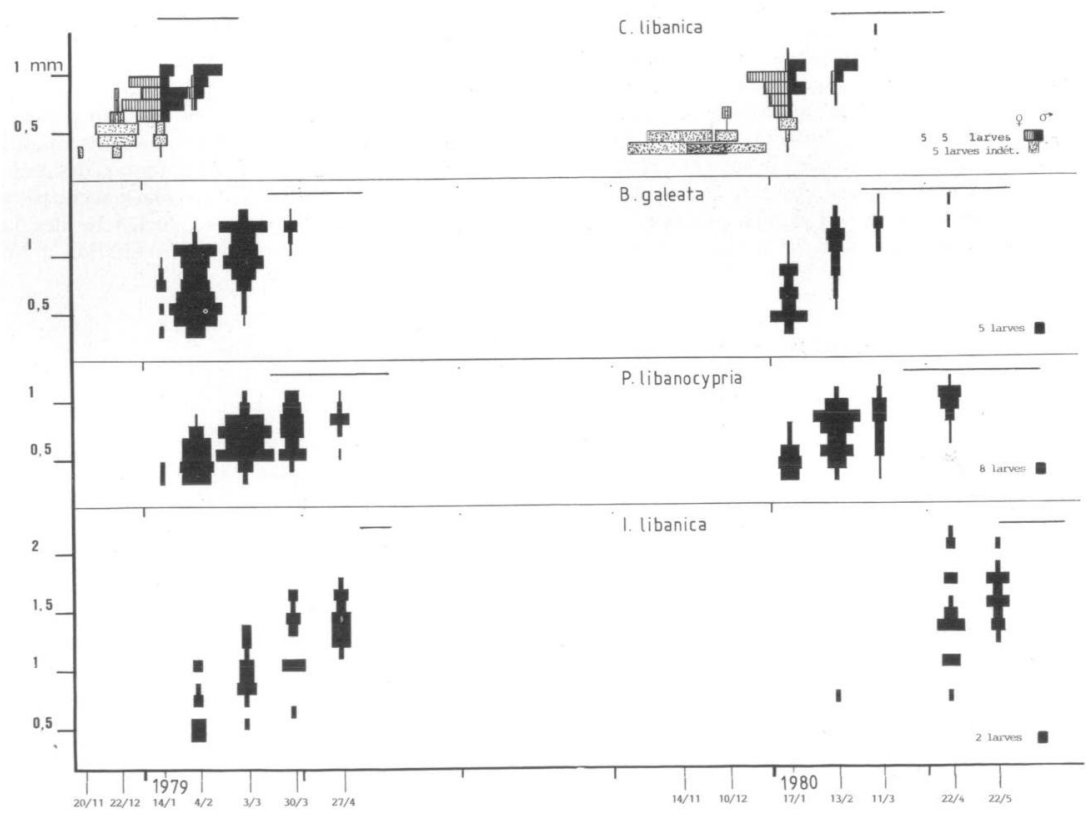

Fig. 1. Développement larvaire et périodes de vol des Plécoptères en 1979 et en 1980 . Les histogrammes représentent les largeurs de la capsule céphalique séparées en classes de $1 / 10 \mathrm{~mm}$. Les larves femelles de Capnia sont celles ayant des fourreaux alaires. Le trait en haut à droite de chaque histogramme représente la période de vol. 


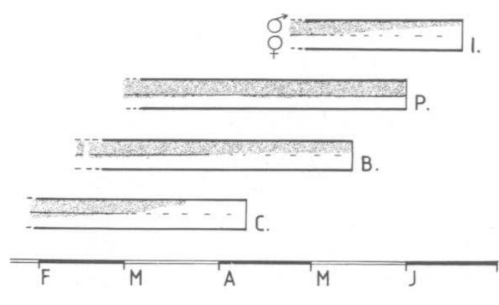

Fig. 2. Périodes de vol de Capnia ? arensi (C.), Brachyptera galeata (B.), Protonemura libanocypria (P.) et lsoperla libanica (I.). Les périodes sont basées sur les récoltes des années 1978, 1979 et 1980. L'espace supérieur en grisé représente les périodes de vol des mâles.

respectives des périodes de vol de $B$. galeata, $P$. libanocypria et $l$. libanica étaient de 9,11 et 3 semaines en 1979 et de 13, 14 et 6 semaines en 1980. La réduction observée en 1979 est en rapport avec la plus faible pluviosité enregistrée cette même année,
$414 \mathrm{~mm} / \mathrm{an}$ pour $867 \mathrm{~mm} / \mathrm{an}$ l'année précédente (Alouf 1983 b). Comme, quelle que soit l'humidité de l'année, l'hiver est toujours pluvicux au Liban, la durée de la période de vol de $C$. ? arensi n'est pas grandement affectée par une faible pluviosité.

\section{Travaux cites}

Alouf (N.J.). 1983 a. - Conlribution à la connaissance des cours d'eau du Liban : La zonation biologique du Nahr Qab Ilias. Annls. Limnol., 19 : 121.127.

Alouf (N.J.) 1983 b. - Cycle de vie de Gammanus laticoxalis ssp. dans l'exsurgence Shtaura (Liban). Note sur Gammarus syriacus de Shamsine. Hydrobiologia, 107 : 169-181.

Aubert (J.). 1964. - Quelques Plécopteres du Muséum d'Histoire Naturelle de Viennc. Ann. Naturh Mus. Wien, 67: 287-301.

Berthélemy (C.). 1979. - Accouplement, période d'incubation et premiers stades larvaires de Brachyptera braueri et de Periodes microcephalus (Plecoptera). Anils. Limnol. 15 : 317-335.

Berthélemy (C.) \& Dia (A.). 1982. - Plécoptères du Liban (Insecta). Annls. Limnol. 18: 191-214.

Légier (P.). 1979. - Recherches sur l'écologie des ruisseaux temporaires. Thèse Ductorat d'etat, Aix-Marseille, $320 \mathrm{p}$.

Saltveit (S.J.) \& Lillehammer (A.). 1984. - Studies on egg development in the Fennoscandian Isoperla species (Plecoptera). Annls. Limnol, 20 : $91-94$.

Zwick (P.). 1978. - Steinlliegen (Insecta, Plecoptera) aus Griechenland und benachbarten Ländern. Mitt. Schweiz Ent. ges., 51 : $21-38,213.239$. 